\title{
A releasable disulfide-linked peptide tag facilitates the synthesis and purification of short peptides
}

Received 00th January 20xx,

\author{
Yuteng Wu, ${ }^{a},{ }^{\dagger}$ Alessandro Zorzi, ${ }^{a}{ }^{+}{ }^{\dagger}$ Jack Williams, ${ }^{a}$ and Christian Heinis*a
}

DOI: $10.1039 / \times 0 \times x 00000 x$

Combinatorial cyclization of hundreds to thousands of random linear peptides by structurally diverse chemical linkers offers access to large macrocyclic compound libraries. A bottleneck in the development of such libraries is the preparation of large numbers of short random linear peptides. Herein, we present a tag-based strategy that is not dependent on a throughput-limiting chromatographic purification step and thus enables parallel production of short peptides. In brief, peptides are synthesized on solid phase as conjugates with a disulfide-linked Cys-Gly-Arg-Trp tetra-peptide tag. The charged arginine residue in the tag allows for purification of the peptides by diethyl ether-precipitation and the tryptophan allows for quantification of the product by absorption measurement. Addition of a reducing agent releases the short peptides from the tag. The released sulfhydryl group in the peptide can readily be used for cyclization of the peptide library with biselectrophilic linker reagents.

Cyclic peptides are an attractive modality for developing binders to challenging targets to which classical small molecule ligands can hardly be developed due to the lack of suitable binding pockets or clefts. ${ }^{1}$ Powerful in vitro display techniques such as phage display or mRNA display have enabled the generation and efficient screening of large combinatorial libraries comprising billions of cyclic peptides and the creation of peptide ligands to a broad range of targets..$^{2,3}$ The techniques have delivered several cyclic peptides that are currently evaluated in clinical trials, most of them addressing extracellular targets. ${ }^{4}$

Many challenges remain when pursuing the class of cyclic peptides as therapeutics, the most important ones being the development of peptide drugs that are cell-permeable or orally available. Both goals require that the cyclic peptides have a rather small molecular mass, ideally well below one $\mathrm{kDa}$, and a

\footnotetext{
a. Institute of Chemical Sciences and Engineering, Ecole Polytechnique Fédérale de Lausanne, CH-1015 Lausanne, Switzerland

+ Authors contributed equally

Electronic Supplementary Information (ESI) available: [details of any supplementary information available should be included here]. See DOI: 10.1039/x0xx00000x
}

limited polar surface, which are both properties that are not met by most peptides derived from phage or mRNA display libraries. In order to synthesize and screen large libraries of small-sized cyclic peptides, various innovative strategies were pursued in recent years, including methods to encode small cyclic peptides by DNA 5,6 and approaches to synthesize cyclic peptides on beads ${ }^{7}$ or in microwell plates. ${ }^{8}$

Our laboratory has recently developed an approach to synthesize and screen large combinatorial libraries of small cyclic peptides in microwell plates. ${ }^{9}$ In brief, a large number of $n$ linear peptides was synthesized and combinatorially cyclized by $m$ bis-electrophile linker reagents to yield $m \times n$ cyclic compounds. Due to the efficient macrocyclization reactions, the products did not require purification, which allowed for screening thousands of small cyclic peptides with a moderate effort. A bottleneck in this approach is the preparation of the random linear peptides. In one study for example, we prepared 168 tripeptides that we combinatorially cyclized with seven biselectrophile linkers to screen a library of 1176 cyclic peptides. ${ }^{9}$ We had to purify all 168 tripeptides by HPLC which was a substantial effort. The HPLC purification was necessary as peptide precipitation by diethyl ether, a process typically applied to remove released protecting groups and cleavage reagents after solid phase peptide synthesis, did not work because the peptides did not precipitate efficiently due to the short length of only three amino acids.

In order to omit an HPLC purification step, we have recently synthesized a library of tetrapeptides carrying a non-cleavable C-terminal Gly-Gly-Trp tag that enabled peptide precipitation by diethyl ether after release from the solid phase, and yielded the peptides at high purity (>95\%). In this way, we generated and screened a library of 8988 cyclic peptides that was based on 1284 tetrapeptides cyclized combinatorially with seven linkers. ${ }^{9}$ The tryptophan residue in the tag allowed for determination of the peptide concentration by absorption measurement. A limitation of the tag was that it biased the activity of some cyclic 
a

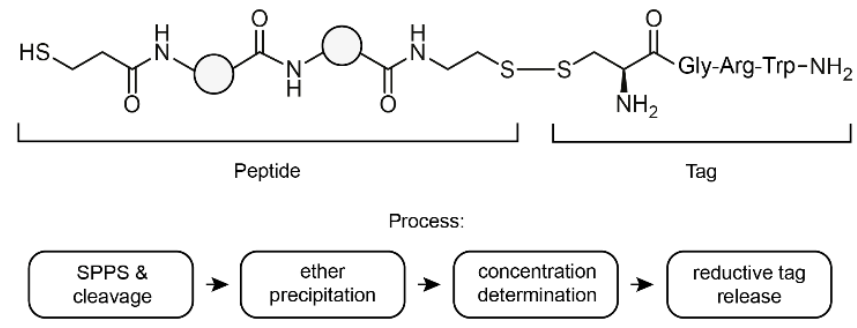

b
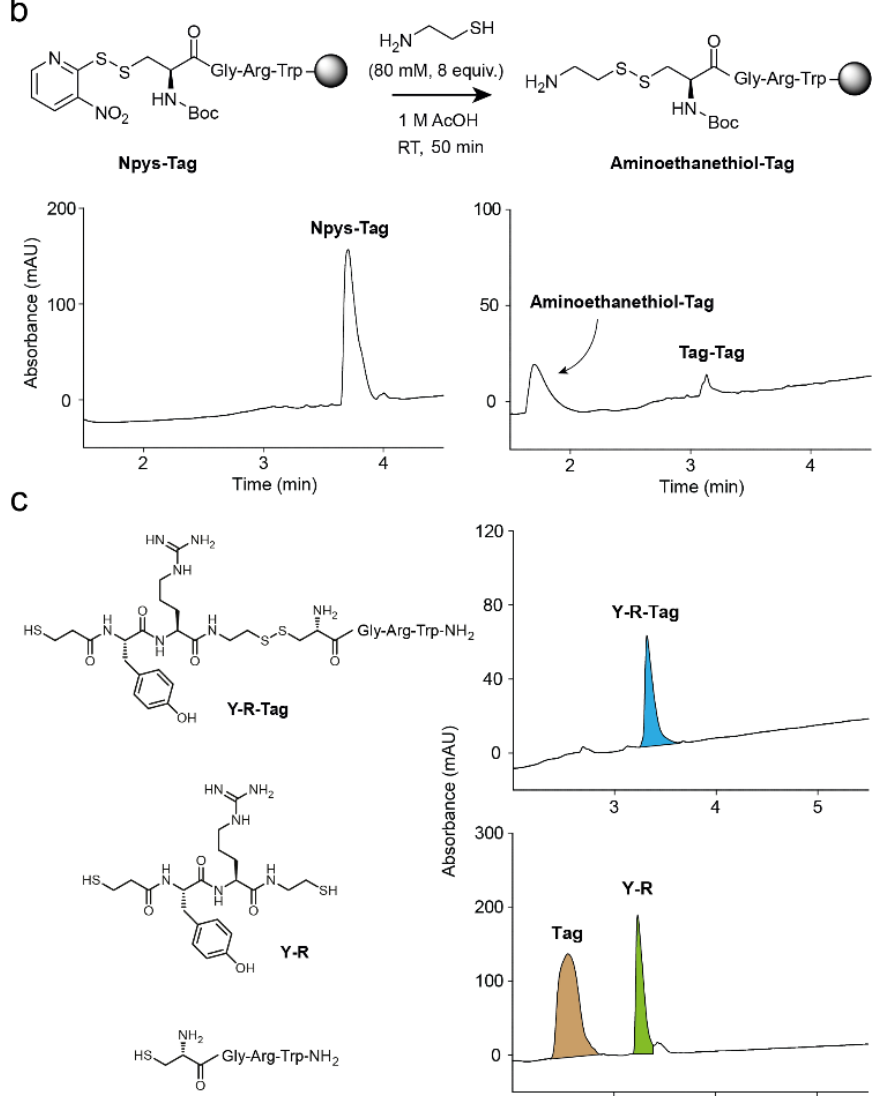

Tag

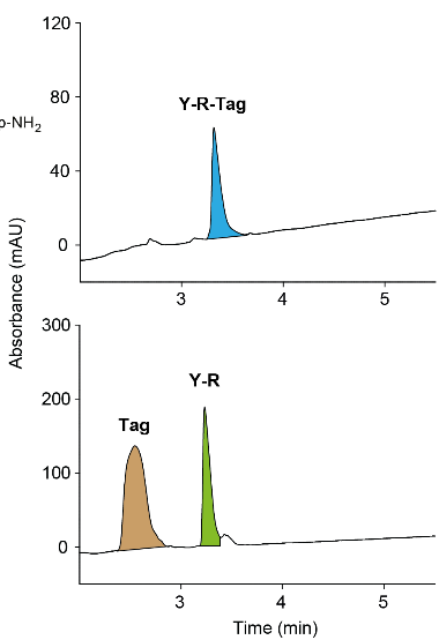

Fig. 1. Strategy of releasable disulfide-linked tag. (a) Chemical structure of peptide linked via a disulfide bridge to the tag. The amino acids represented as round circles can be varied in peptide libraries. The positively charged arginine residue in the tag allows for efficient precipitation by diethyl ether. The trypthophan residue enables quantification of peptide yields by absorption measurement. The experimental steps to generate free peptide at high purity are described. (b) Solid-phase peptide synthesis of disulfide-linked tag. The disulfide bridge is introduced via a disulfide exchange reaction. The Npys group in the Boc-Cys(Npys)-OH amino acid is a good leaving group and allows for efficient disulfide exchange. The left absorption chromatogram shows the LC-MS analysis of Boc-Cys(Npys)-GlyArg-Trp (Npys-Tag) after cleavage from resin. The right chromatogram shows analysis of the tag after the disulfide exchange reaction (Cysteamine-Tag). (c) Tagged model peptide $\mathrm{Y}-\mathrm{R}$-Tag and reductive release of the tag by two equiv. of TCEP to obtain the free peptide $Y-R$. peptides during the library screening because it contributed to the binding. For example the best hits identified in the screen against tissue kallikrein 5, R-75-20-C-GGW-6 $\left(K_{\mathrm{i}}=144 \mathrm{nM}\right)$ and R-75-G-C-GGW-6 $\left(K_{\mathrm{i}}=610 \mathrm{nM}\right)$ showed a 139- and 36-fold lower target inhibition when the Gly-Gly-Trp tag was removed. ${ }^{9}$

Herein, we aimed at developing a strategy for the efficient parallel synthesis and purification of large numbers of short peptides that avoids the problems described above. We envisioned an approach in which peptides are synthesized with a tag that enables purification by precipitation with diethyl ether and measurement of the peptides' concentrations by UV light absorption, wherein the tag can be released easily prior to the peptide-binding screen for ensuring that the tag does not bias the target binding. We conceived the peptide-tag format shown in Figure 1a, in which peptide and tag are linked by a reducible disulfide bridge. Connection via a disulfide bridge was attractive because the bond could be cleaved efficiently by addition of a small molar excess of reducing agent and because the resulting thiol group could be used directly as handle for cyclizing the peptides. The small molar excess of reducing agent over peptide was expected to not interfere substantially with the peptide cyclization reactions. The reducing agent was expected to neither impose a problem for most activity screens such as enzyme inhibition assays or fluorescence-based proteinprotein interaction assays. It was our intention to not purify the peptides from the tag after tag release and to perform both, the chemical cyclization of the peptides and the biological screens, in presence of the released tag, as we wanted to omit a purification step. We expected that a small tag would not interfere with the screening assay if it was present but not linked to the peptides.

We designed the tag to contain i) a cysteine for forming a disulfide bridge with the library peptides, ii) an arginine and thus a charged side chain for efficient precipitation of the peptides by diethyl ether for purification after release of peptides from the solid phase, and iii) a tryptophan residue for measuring the peptide concentration. The measurement of the concentration offered the option to adjust the peptide concentrations in case the synthesis yields were varying strongly for the different peptides in the library. We chose to synthesize a tag with the sequence Cys-Gly-Arg-Trp which is linked to a thiol group at the C-termini of the library peptides, as shown in Figure $1 a$.

We developed the following solid-phase peptide synthesis strategy for generating tagged library peptides. The tag with the sequence Cys-Gly-Arg-Trp was first synthesized on rink-amide resin using standard Fmoc solid-phase peptide synthesis conditions, a disulfide bridge was then established between the cysteine and the $\mathrm{C}$-terminal building block of the library peptide (cysteamine), and the library peptide was then appended using again standard Fmoc solid-phase synthesis conditions (Fig. 1a). The disulfide bridge was established using the cysteine building block Boc-Cys(Npys)-OH that carried a 3-nitro-2-pyridylsulfenyl (Npys) thiol-protecting group (Fig. 1b). In addition to being a protecting group, Npys is a good leaving group for disulfide 
a

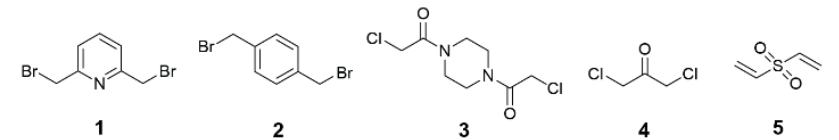

b

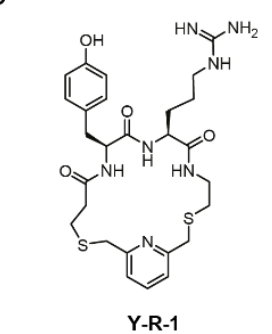

C

\begin{tabular}{cc} 
linker: & $\begin{array}{c}\text { macrocycle } \\
\text { yield }(\%):\end{array}$ \\
\hline 1 & 97 \\
2 & 53 \\
3 & 71 \\
4 & 100 \\
5 & 96
\end{tabular}

d

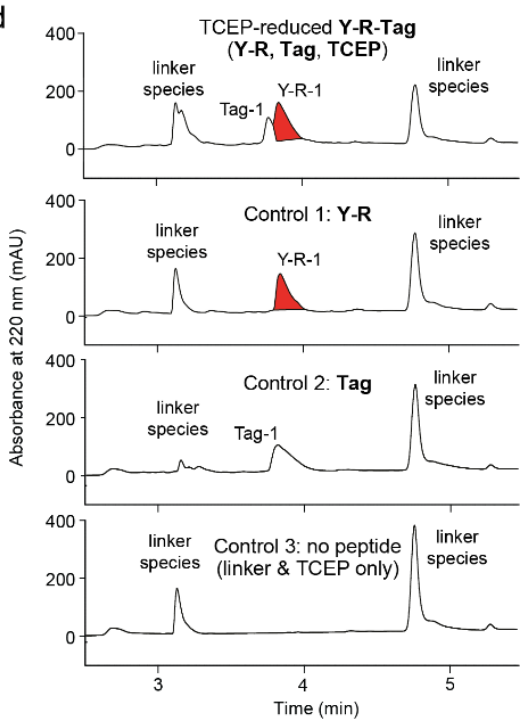

Fig. 2. Macrocyclization of short peptides. (a) Bis-electrophilic reagents for the cyclization of peptides via two thiol groups. (b) Chemical structure of peptide Y-R cyclized with reagent 1 . (c) Yields obtained for the cyclization of the peptide $Y-R$ with the reagents 1-5. (d) Chromatograms of LC-MS analysis of the macrocyclization of TCEP-reduced Y-R-Tag with reagent $\mathbf{1}$ (top chromatogram). The peak of the desired product $\mathrm{Y}-\mathrm{R}-1$ is highlighted in red. The other three chromatograms show the analysis of control reactions: reaction of purified peptide $Y-R$ with $\mathbf{1}$, reaction of purified Tag with $\mathbf{1}$, and reaction reagents without peptide.

exchange reactions. ${ }^{10}$ It has been used for the synthesis of disulfide-cyclized peptides on solid phase and in solution, ${ }^{11}$ disulfide-conjugation of peptides to other peptides and to proteins, ${ }^{12}$ and for the linkage of peptides to resin via a disulfide bridge. ${ }^{12,13}$ We chose to synthesize the peptides on PEGA resin due to its compatibility with aqueous solvents and reaction at low $\mathrm{pH}$, which is suited for an efficient disulfide exchange reaction. ${ }^{12,14}$

We first tested the synthesis of Boc-Cys(Npys)-Gly-Arg-Trp (Npys-Tag) on PEGA resin and found that it was obtained with > $90 \%$ purity (Fig. 1b, left chromatogram). We then treated the resin-bound Npys-Tag with cysteamine ( $80 \mathrm{mM}, 8$ equiv.) under acidic conditions ( $1 \mathrm{M} \mathrm{AcOH}$, around $\mathrm{pH} 2$ ) to generate Cysteamine-Tag conjugate via an intermolecular disulfide exchange reaction (Fig. 1b). Cleavage of the product from the rink-amide resin $(95 \%$ trifluoroacetic acid, $2.5 \%$ triisopropylsilane, 2.5\% water) and LC-MS analysis revealed that around $90 \%$ of the tag was modified with cysteamine and only a small fraction of the tag remained free, wherein the free tag eluted as the disulfide-linked tag dimer (Tag-Tag; Fig. 1b, right chromatogram). We reasoned that the free tag would not be a limitation for the approach because we expected that the short peptide does not interfere with the screening assays, as described above.

We next appended to the free amino group the model dipeptide Tyr-Arg and an N-terminal 3-mercaptopropionic acid (MPA, carrying a Trt thiol-protecting group) using standard Fmoc solid-phase peptide synthesis reagents and conditions. The additional thiol group at the $\mathrm{N}$-terminus was introduced to enable thiol-to-thiol macrocyclization of the model peptide by bis-electrophile reagents. Cleavage of the product $(95 \%$ trifluoroacetic acid, $2.5 \%$ triisopropylsilane, $2.5 \%$ water) and precipitation by diethyl ether yielded the desired product termed $\mathrm{Y}-\mathrm{R}$-Tag, and as expected, a small fraction of free tag. If the free tag was excluded as impurity, the purity of the Y-R-Tag was $>95 \%$ based on the chromatographic analysis. Despite the high purity and our plans to not purify the peptides chromatographically in future, we purified the Y-R-Tag by HPLC to more easily study the next reaction step, the reductive release of the tag. Incubation of the HPLC-purified Y-R-Tag model peptide $(6.7 \mathrm{mM})$ with a 2 -fold molar excess of the reducing agent TCEP (tris-carboxyethyl-phosphine) in DMSO and incubation for $30 \mathrm{~min}$ at room temperature led to facile release of the peptide, as assessed by LC-MS analysis (Fig. 1c, lower panel).

We next tested if the released peptide $Y-R$, in presence of the released tag and the reducing agent TCEP, can be cyclized with bis-electrophilic linker reagents. We tested the structurally diverse reagents 1-5 shown in Figure 2a. The cyclic peptide product $\mathrm{Y}-\mathrm{R}-1$ expected for the reaction with reagent $\mathbf{1}$ is shown in Figure $2 \mathrm{~b}$. All five reagents were previously found to efficiently cyclize pairs of cysteines in peptides. ${ }^{15}$ We incubated Y-R-Tag $(6.7 \mathrm{mM})$ with two equiv. TCEP $(13 \mathrm{mM})$ in DMSO to release the $\mathrm{Y}-\mathrm{R}$ peptide, added the products to the reagents 1 5 dissolved in ammonium carbonate buffer and DMSO, and incubated the reactions for $1 \mathrm{hr}$ at room temperature. The final concentrations and solvent fractions were $0.33 \mathrm{mM} \mathrm{Y-R-Tag,}$ $0.67 \mathrm{mM}$ TCEP, $2 \mathrm{mM}$ reagent 1-5, 15\% DMSO, and 85\% $60 \mathrm{mM}$ $\mathrm{NH}_{4} \mathrm{HCO}_{3}$ buffer, $\mathrm{pH}$ 8. LC-MS analysis showed that the $\mathrm{Y}-\mathrm{R}$ peptide was efficiently released and cyclized by all linkers with yields above $50 \%$ (Fig. 2c). Chromatograms of the reactions are shown in Figure $2 \mathrm{~d}$ for the cyclization with reagent $\mathbf{1}$ and in Figure S1 for the other reagents. To identify more easily the reaction products and side products, we performed control reactions in which Y-R peptide only (purified), Tag only (purified) and no peptide were incubated with the bis-electrophile reagents (Fig. 2d and Fig. S1).

To test if the tag-based strategy is suited for preparing a larger number of short linear peptides, we synthesized 81 random peptides of the format shown in Figure 3a. The peptides contained an N-terminal thiol group, a first (A) and second amino acid (B) selected from the nine building blocks shown in Figure $3 \mathrm{a}$, and the $\mathrm{C}$-terminal cysteamine group. Five of the nine amino acids chosen were unnatural and two of the latter group were not $\alpha$-amino acids, yielding peptides with structurally diverse backbones. We first prepared PEGA resin carrying the Boc-Cys(Npys)-Gly-Arg-Trp tag on a larger scale (1.5 g). Following the disulfide exchange reaction, we added $16 \mathrm{mg}$ of 
a<smiles>O=C(CCS)NCC(=O)NCC(=O)NCCS</smiles>

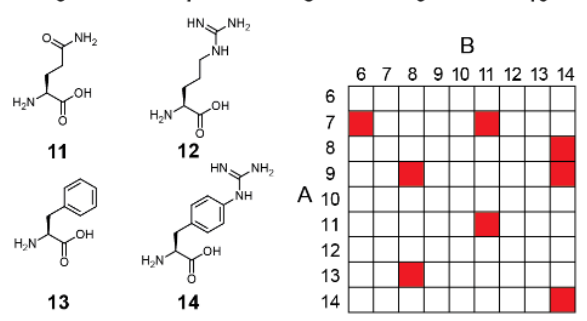

b

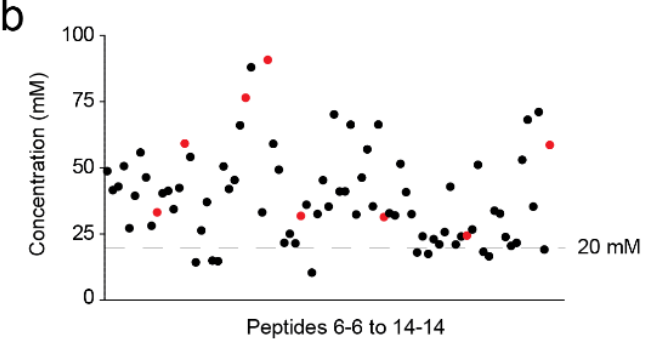

Peptides 6-6 to $14-14$
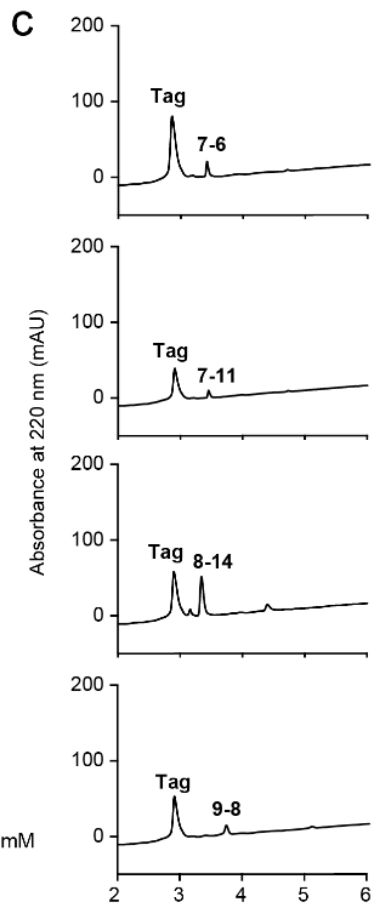
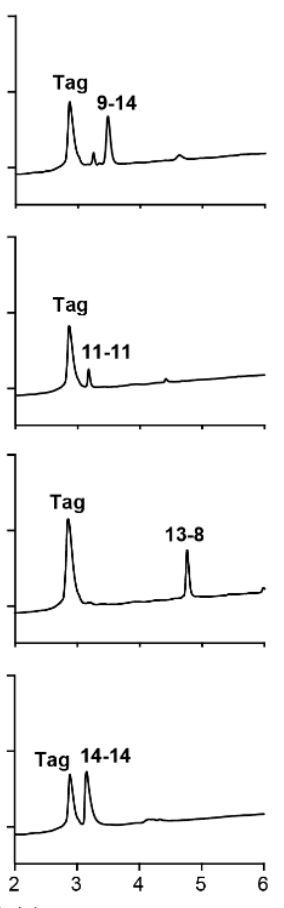

one inhibiting thrombin with a $K_{i}$ of $17 \mu \mathrm{M}$ (cyclic peptide 1 ), and one with a $K_{\mathrm{i}}$ of $\sim 1 \mathrm{mM}$ (cyclic peptide 2) (Figure 4a and Figure S2). We chose on purpose peptides displaying rather weak inhibitory activities as measuring such activities in the presence of potential impurities is more challenging. We synthesized the two peptides using the tag-based strategy, purified them by precipitation with diethyl ether, released the tag, cyclized the crude peptides by bis-electrophile linker reagent 1 (Figure S3), and measured thrombin inhibition. The cyclic peptide 1 inhibited around $40 \%$ of the thrombin activity, which was expected for the concentration used, and the cyclic peptide 2 showed only weak inhibition, also as expected (around 10\% inhibition; Figure 4b). The good correlation of the activity of peptides produced with the tag-based approach and HPLC-purified peptides suggests that the impurities do not interfere with the bioassay. Importantly, the results show that the tag, which is also present in the activity assay, does not pose a problem.

a

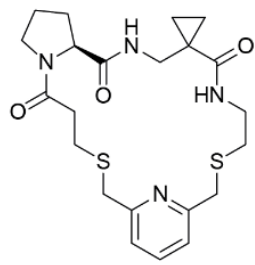

cyclic peptide 1

$K_{\mathrm{i}}=17 \pm 1 \mu \mathrm{M}$

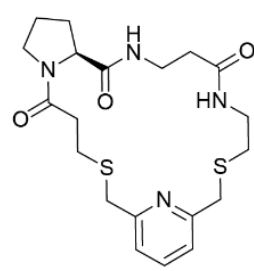

cyclic peptide 2

$K_{\mathrm{i}} \sim 1 \mathrm{mM}$

and the concentrations determined by measuring absorption of the tryptophan in the tag. Of the 81 peptides, 72 displayed concentrations above $20 \mathrm{mM}$ (around 90\%; Fig. 3b). We randomly selected eight peptides for purity analysis (indicated in red in Fig. 3a). All eight peptides displayed purities of $>50 \%$ if Tag-Tag peak was included, and $>70 \%$ if the Tag-Tag peak was excluded, as assessed by LC-MS. Application of previously established reduction conditions with TCEP (two equiv.) resulted in a full conversion to the short peptides and tag (Fig. 3c). All peptides displayed a high purity as seen in the chromatograms in Figure $3 \mathrm{c}$ in which absorption was recorded at $220 \mathrm{~nm}$, to see the peptides and potential impurities.

Finally, we tested if impurities such as the released tag could interfere in a bioassay, which would be a problem as it could hinder or even prevent the screening of the non-HPLC-purified peptides. As example for a typical therapeutic target and screening assay, we chose thrombin and the measurement of its inhibition in a 384-microwell plate assay with a fluorogenic substrate. As standard peptides, we used two cyclic peptides,

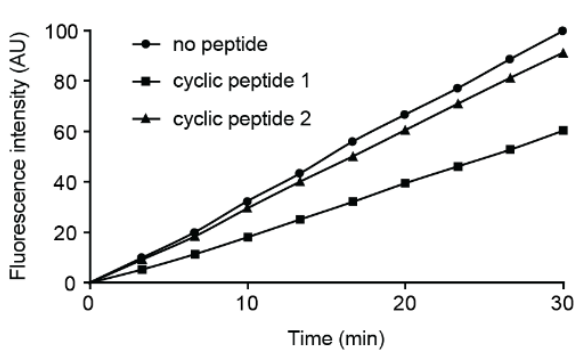

Fig. 4. Measuring activity of cyclic peptides produced with the tag-based strategy. (a) Standard peptides inhibiting thrombin with weak affinities. The $K_{\mathrm{i}} \mathrm{S}$ were determined using HPLCpurified peptides. (b) Residual thrombin activity in presence of cyclic peptides produced with the tag-based strategy (not HPLC purified). Thrombin activity was measured with a fluorogenic substrate. 
In conclusion, we have established an approach for the efficient generation of large numbers of short peptides. Synthesis of the peptides as conjugates with a precipitable tag allowed for purification of the peptides to a high degree and determination of the concentrations by absorption measurement. Conjugation of the tag via a disulfide bridge allowed for efficient release by addition of only two equivalents of the reducing agent TCEP. We showed that the liberated thiol group in the peptides can directly be used for peptide cyclization by diverse bis-electrophile linker reagents. In a proof of concept, we demonstrated that the approach is applicable to random peptide sequences and yields short peptides of remarkable purity, in particular when considering the omission of a chromatographic purification step. We envision that the strategy is applicable for the generation of large libraries of short linear peptides. Combinatorial cyclization of such peptides with structurally diverse linker reagents will offer access to large and structurally highly diverse cyclic peptide libraries.

\section{Conflicts of interest}

There are no conflicts to declare.

\section{References}

1 E. Valeur, S. M. Guéret, H. Adihou, R. Gopalakrishnan, M. Lemurell, H. Waldmann, T. N. Grossmann and A. T. Plowright, Angew. Chemie Int. Ed., 2017, 56, 10294-10323.

2 K. Deyle, X.-D. Kong and C. Heinis, Acc. Chem. Res., 2017, 50, 1866-1874.

3 Y. Huang, M. M. Wiedmann and H. Suga, Chem. Rev., 2019, 119, 10360-10391.

4 A. Zorzi, K. Deyle and C. Heinis, Curr. Opin. Chem. Biol., 2017, 38, 24-29.

5 D. R. Gartner, Z. J., Tse, B.N., Grubina R., Doyon J.B., Snyder, T.M., Liu, Science (80-. )., 2004, 305, 1601-1605.

6 Y. Li, R. De Luca, S. Cazzamalli, F. Pretto, D. Bajic, J. Scheuermann and D. Neri, Nat. Chem., 2018, 10, 441-448.

7 Z. Guo, S. Y. Hong, J. Wang, S. Rehan, W. Liu, H. Peng, M. Das, W. Li, S. Bhat, B. Peiffer, B. R. Ullman, C.-M. Tse, Z. Tarmakova, C. Schiene-Fischer, G. Fischer, I. Coe, V. O. Paavilainen, Z. Sun and J. O. Liu, Nat. Chem., 2019, 11, 254-263.

8 D. Bernhagen, V. Jungbluth, N. G. Quilis, J. Dostalek, P. B. White, K. Jalink and P. Timmerman, ACS Comb. Sci., 2019, 21 198-206.

9 S. S. Kale, M. Bergeron-Brlek, Y. Wu, M. G. Kumar, M. V. Pham, J. Bortoli, J. Vesin, X.-D. Kong, J. F. Machado, K. Deyle, P. Gonschorek, G. Turcatti, L. Cendron, A. Angelini and C. Heinis, Sci. Adv., 2019, 5, eaaw2851.

10 R. MATSUEDA and R. WALTER, Int. J. Pept. Protein Res., 2009, 16, 392-401.

11 C. Rentier, K. Fukumoto, A. Taguchi and Y. Hayashi, J. Pept. Sci., 2017, 23, 496-504.

12 B. Ponsati, M. Ruiz-Gayo, E. Giralt, F. Albericio and D. Andreu, J. Am. Chem. Soc., 1990, 112, 5345-5347.

13 W. Tegge, W. Bautsch and R. Frank, J. Pept. Sci., 2007, 13, 693699.

14 M. S. BERNATOWICZ, R. MATSUEDA and G. R. MATSUEDA, Int. J. Pept. Protein Res., 2009, 28, 107-112.

15 S. S. Kale, C. Villequey, X.-D. Kong, A. Zorzi, K. Deyle and C. Heinis, Nat. Chem., 2018, 10, 715-723.

\section{Graphical Abstract}

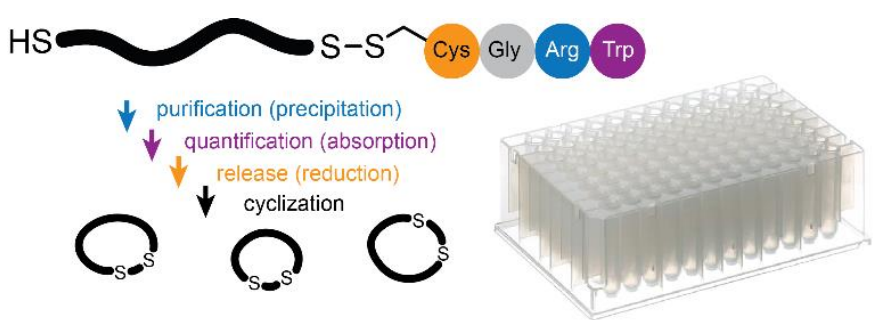

